\title{
THE TURBULENT INTERSTELLAR MEDIUM AND PRESSURE-BOUNDED MOLECULAR CLOUDS
}

\author{
Philip Maloney \\ Department of Planetary Sciences, University of Arizona, and Sterrewacht Leiden \\ Received 1987 February 17; accepted 1988 May 10
}

\begin{abstract}
The existence of turbulence throughout the interstellar medium suggests that an appropriate value for the average pressure may be $P / k \gtrsim 10^{4}$. Negative-index polytropic models of interstellar clouds in equilibrium with an external medium at these pressures are predicted to have sizes, line widths, masses, and size-line width and size-density relations in good agreement with those observed and inferred for dark clouds. Thus these observed features of interstellar clouds do not require that they be completely self-gravitating or "virialized" in the commonly used sense.
\end{abstract}

Subject headings: interstellar: matter - interstellar: molecules - turbulence

\section{INTRODUCTION}

One of the major goals of molecular astronomy has been to understand the physics and dynamics of molecular clouds. It has generally been assumed, and occasionally claimed to have been conclusively demonstrated, that molecular clouds (with the exception of the diffuse clouds) are gravitationally bound and in virial equilibrium. (The term "virial equilibrium" in reference to molecular clouds is commonly used to mean that the line width is directly related to the cloud mass and radius as given by the virial theorem in the absence of any surface pressure or magnetic field terms.) However, the realization that the average pressure in the ISM is probably dominated by the contribution of turbulence suggests that another scenario is possible, wherein a significant fraction of molecular clouds are in pressure equilibrium with an intercloud medium with $P / k \approx 2 \times 10^{4}$ and are not dominated by self-gravity. In $\S$ II I discuss the evidence for high pressures in the ISM, in $\S$ III I derive the properties of pressure-bounded clouds, and in $\S$ IV I compare the model cloud properties with observations. Finally, in $\S$ V I discuss some of the implications of the existence of such clouds in the ISM.

\section{TURBULENT PRESSURE IN THE ISM}

Determinations of pressure in the ISM have relied on estimates of particle densities and temperatures in diffuse interstellar clouds. The most direct measurements have used observations of molecular hydrogen (Jura 1975) and the C I fine-structure lines (Jenkins and Shaya 1979; Jenkins, Jura, and Loewenstein 1983$)$ in cold clouds $\left(T_{k} \lesssim 100 \mathrm{~K}\right)$. Both data sets indicate the presence of a wide range of pressures in the ISM; Jenkins et al. find $P / k$ ranges from less than $10^{3}$ to more than $10^{5} \mathrm{~cm}^{-3} \mathrm{~K}$ for different lines of sight. However, these values refer solely to the microscopic, thermal component of the pressure. The line widths measured in these studies are far larger than thermal Doppler widths, even when saturation is taken into account, and demonstrate that turbulence is the dominant line-broadening mechanism. The Doppler $b$-values inferred from the line widths are typically $1-2 \mathrm{~km} \mathrm{~s}^{-1}$.

These results, namely, that turbulence is widespread and typical values of $b_{\text {turb }}$ are $1 \mathrm{~km} \mathrm{~s}^{-1}$ or more, are supported by a large body of data on the ISM. Hobbs's (1974) high-resolution interferometric study of optical absorption lines of $\mathrm{K} \mathrm{I}$, Na I, and $\mathrm{Ca}$ II produced an average value of $b=1.49 \mathrm{~km} \mathrm{~s}^{-1}$ for a sample of 30 clouds. A much more estensive study of $\mathrm{K}$ I absorption lines by Chaffee and White (1982) yielded $b$ values of $1-2 \mathrm{~km} \mathrm{~s}^{-1}$ for single clouds. Since the abundance of neutral species such as $\mathrm{K}$ I depends so strongly on density, these studies must sample the densest, most localized clouds in the diffuse ISM. Well-determined $b$ values for optical absorption lines of $\mathrm{CN}, \mathrm{CH}$, and $\mathrm{CH}^{+}$(Crutcher 1985; van Dishoeck and Black 1987; Hawkins, Jura, and Meyer 1986; Meyer and Jura 1985) for various diffuse clouds and one dark cloud range from $1-2 \mathrm{~km} \mathrm{~s}^{-1}$

In a medium where both turbulence and thermal gas motions contribute to the pressure, it is given by

$$
P / k=\sum_{i} n_{i} T+(\delta v)^{2} \rho / 2 k \mathrm{~cm}^{-3} \mathrm{~K}
$$

(van Dishoeck and Black 1986), where $\delta v=10^{5} b$ is the Gaussian velocity dispersion in $\mathrm{cm} \mathrm{s}^{-1}$ which characterizes the turbulence, and the summation is over the constituents of the gas with densities $n_{i}$ in $\mathrm{cm}^{-3}$; these are mainly $\mathrm{H}, \mathrm{H}_{2}$, and $\mathrm{He}$. Including a turbulent contribution to the pressure with $b=1.5 \mathrm{~km} \mathrm{~s}^{-1}$ would raise the median value for $P / k$ of Jenkins, Jura, and Loewenstein (1983) from $4.0 \times 10^{3}$ to $1.3 \times 10^{4}$, assuming $T=80 \mathrm{~K}$. Some lines of sight have $P / k$ considerably in excess of $10^{5}$ when this turbulent contribution is included.

The detailed cloud models of van Dishoeck and Black (1986) also suggest that the pressure in the intercloud medium must be substantially larger than inferred from temperatures and densities. Their models, which successfully reproduce a large amount of molecular line data for the clouds in front of $\zeta$ Per, $\zeta$ Oph, $\chi$ Oph, and $o$ Per, have surface pressures ranging from $P / k \approx 10^{4}$ to $4 \times 10^{5}$. Although they assumed a polytropic equation of state, rather than calculating thermal balance explicitly, it is unlikely that the surface pressures are greatly in error. If one is to avoid the conclusion that diffuse clouds are expanding rapidly, then the intercloud pressure must be of the order of $10^{4} \mathrm{~cm}^{-3} \mathrm{~K}$, on average. Since turbulence is so widespread in the ISM on a variety of scales, it is reasonable to expect that pressure in the intercloud medium also has a large contribution from turbulent motion. 


\section{PROPERTIES OF PRESSURE-BOUNDED CLOUDS}

Calculations of the equations of state for interstellar cloud models assuming local thermal balance (Viala 1972; Shu et al. 1972; de Jong, Dalgarno, and Boland 1980) indicate that negative-index polytropes with $P=K \rho^{1+1 / N}$ provide reasonable approximations for a range of values of the polytropic index, $N$. For a full discussion of the properties and stability of negative-index polytropes, see Viala and Horedt (1974b). Here I derive the properties of spherical polytropic cloud models that are bounded by an external medium with pressure $P_{e}$. I will use the usual polytropic variables $\theta$ and $\xi$. The values of $\xi$ and $\theta$ at the polytrope boundary will be denoted by $\xi_{e}$ and $\theta_{e}$.

For a polytrope in equilibrium with an external pressure $P_{e}$, the polytropic constant can be determined from

$$
K=\frac{P_{e}}{\rho_{0}^{1+1 / N} \theta_{e}},
$$

where $\rho_{0}$ is the central density. The physical radius of the polytrope is related to the dimensionless length variable $\xi_{e}$ by

$$
\frac{r_{e}}{\xi_{e}}=\left[\frac{-(N+1) K}{4 \pi G} \rho_{0}^{1 / N-1}\right]^{1 / 2} .
$$

Viala and Horedt (1974b) showed that pressure-bounded polytropes with $n<-1$ are unstable if $\xi$ is larger then a critical value, $\xi_{c}(N)$ : an increase in the bounding pressure $P_{e}$ will cause the polytrope to collapse. However, as was first pointed out by Dickman and Clemens (1983), this instability depends on the assumption that the polytropic constant $K$ is constant for the sequence of polytropes produced by varying the external pressure. The general uniformity of gas temperatures observed in molecular clouds over a large range of spatial and density scales (e.g., Leung 1985) suggests that a more appropriate boundary condition for models of interstellar clouds is probably one where the central temperature $T_{c}$ of the polytrope remains constant when the boundary pressure is varied. It is straightforward to demonstrate that polytropes with $N<-1$ are stable for all values of $\xi$ when the central temperature is kept fixed (see Appendix). The boundary value of $\xi$ can thus be chosen arbitrarily; the resulting polytropes have nearly uniform densities for small $\xi(<1)$ and are strongly centrally condensed for large $\xi(\gtrsim 10)$.

Equations (2) and (3) and the expression for the mean density of a polytrope, $\bar{\rho}=3 \rho_{0} \theta_{e}^{\prime} / \xi_{e}$, yield a relation between mean density and cloud radius:

$$
\bar{\rho}=1.25 \times 10^{-23}\left[\frac{-(N+1)}{\theta_{e}^{n+1}}\left(\frac{P_{e}}{k}\right)\right]^{1 / 2} \theta_{e}^{\prime} r_{e}^{-1} \mathrm{~g} \mathrm{~cm}^{-3},
$$

where $r_{e}$ is in parsecs. It is easy to show that the mean density is not very sensitive to the boundary value of $\xi$, provided that $\xi_{e} \gtrsim 1$. Thus the mean cloud density varies inversely with radius.

The polytropic temperature here represents the contributions of both thermal and turbulent motions to the support of the cloud. Therefore the scaling of line width with radius is determined by the scaling of polytropic temperature with size. Using equations (2) and (4) to express the central density in terms of $P_{e}$ and $r_{e}$, we can write the temperature at the center of the polytrope as

$$
T_{0}=\frac{\mu r_{e}}{R \xi_{e}}\left[\frac{4 \pi G}{-(N+1)} \frac{P_{e}}{\theta_{e}^{N+1}}\right]^{1 / 2} \mathrm{~K}
$$

where $R$ is the gas constant and $\mu$ is the molecular weight of the gas $\left(\mathrm{g} \mathrm{mol}^{-1}\right)$, equal to 2.25 for standard abundances. The turbulent contribution to the line width is independent of mass; we will calculate the one-dimensional velocity dispersion, $\sigma_{v}=(k T / m)^{1 / 2}$ for the mean particle mass, $\bar{m}=8 / 7 m_{\mathrm{H}_{2}}$. The velocity dispersion is then

$$
\sigma_{v_{0}}=\left\{\frac{\mu k}{R \bar{m}} \frac{r_{e}}{\xi_{e}}\left[\frac{4 \pi G}{-(N+1)} \frac{P_{e}}{\theta_{e}^{N+1}}\right]^{1 / 2}\right\}^{1 / 2} \mathrm{~cm} \mathrm{~s}^{-1},
$$

where the subscript 0 denotes the cloud center value of $T$. For a Gaussian line, the FWHM $\Delta V=(8 \ln 2)^{1 / 2} \sigma_{v}$. Since $T=$ $T_{0} \theta$, the calculated line width increases with increasing radius, scaling as $\theta^{1 / 2}$. The cloud edge line width $\Delta V_{e}$ may be 2 or 3 times larger than $\Delta V_{0}$ for large $\xi_{e}(\approx 20)$ (see discussion in $\S \mathrm{V}$ ).

At low polytropic temperatures, where the thermal component of motion begins to dominate, equation (6) will overestimate the observable line widths for molecules such as $\mathrm{CO}$ and $\mathrm{NH}_{3}$. This also assumes the line is optically thin; for optically thick lines the observable line width will be larger by some factor $\alpha_{s}$ which depends on the degree of saturation. Inserting numerical values into equation (6) yields a line FWHM

$$
\Delta V_{0}=1.35 \times 10^{-1} \alpha_{s} \frac{r_{e}^{1 / 2}}{\xi_{e}^{1 / 2}}\left[\frac{P_{e} / k}{-(N+1) \theta_{e}^{N+1}}\right]^{1 / 4} \mathrm{~km} \mathrm{~s}^{-1}
$$

The value of $\Delta V_{0}$ is rather insensitive to the value of the external pressure, and to the actual value of $\xi_{e}$ for $\xi_{e} \gtrsim 1$. For example, for an $N=-3$ polytrope and $P_{e} / k=10^{4}, \Delta V_{0}=$ $0.78 \alpha_{s} r_{e}^{1 / 2} \mathrm{~km} \mathrm{~s}^{-1}$ for $\xi_{e}=5$, while for $\xi_{e}=20, \Delta V_{0}=$ $0.56 \alpha_{s} r_{e}^{1 / 2} \mathrm{~km} \mathrm{~s}^{-1}$. The spread in $\Delta V$ produced by varying $N$ between -1.2 and -10 is smaller than the range in observed $\Delta V$ at a given radius. Thus an ensemble of clouds with $\xi_{e} \gtrsim 1$ will show size-line width scaling that is indistinguishable within the observational scatter from a simple $\Delta V \propto r_{e}^{1 / 2}$ relation.

\section{COMPARISON WITH OBSERVATIONS}

Studies of dark clouds and dark cloud cores (Leung, Kutner, and Mead 1982; Myers 1983) have suggested that there is a correlation of line width with cloud size of the form $\Delta V \propto r^{1 / 2}$ and between mean number density (as inferred either from detailed excitation calculations or LTE analysis) and cloud size of the form $\bar{n}=r^{-1}$. As has been shown above, these relations are predicted from models of pressure-bounded clouds. Before comparing the model results to the data, however, it is necessary to reexamine some of the observations of dark clouds, since a number of complicating factors render the earlier analyses suspect.

Myers (1983) used ammonia data from Myers and Benson (1982) and CO data from Leung, Kutner, and Mead (1982; hereafter LKM) to derive the relations discussed above for cloud size, mean density, and line width, and found a continuity between the dense, dark cloud cores studied in ammonia and the larger, isolated dark globules observed by LKM. Reanalysis of the LKM data, however, suggests that the dense cloud cores are quite distinct objects in terms of their dominant physics. LKM derived cloud masses from an expression that depends on the maximum column density through the cloud. They used LTE analysis of ${ }^{12} \mathrm{CO}$ and ${ }^{13} \mathrm{CO}$ observations to estimate ${ }^{13} \mathrm{CO}$ column densities; the $\mathrm{H}_{2}$ column density was then obtained by multiplying by the value of $\left[\mathrm{H}_{2}\right] /\left[{ }^{13} \mathrm{CO}\right]$. However, they used a value for this quantity of $10^{7}$, which is a 
factor of 20 larger than the widely used value derived by Dickman (1978). More to the point, current estimates of $\left[{ }^{12} \mathrm{CO}\right] /\left[\mathrm{H}_{2}\right]$ in fully molecular clouds $\left(\gtrsim 8 \times 10^{-5}\right.$; Black and Willner 1984) and of $\left[{ }^{12} \mathrm{CO}\right] /\left[{ }^{13} \mathrm{CO}\right](\approx 45$; Jura 1987) make the adoption of such a large value of $\left[\mathrm{H}_{2}\right] /\left[{ }^{13} \mathrm{CO}\right]$ unjustifiable. Thus the cloud masses (and the mean densities) obtained by LKM are probably too high by a factor of $\sim 20$.

There are also serious disagreements between the data of LKM and those of Martin and Barrett (1978), who observed a number of the same clouds using the same telescope (the NRAO $11 \mathrm{~m}$ ). Martin and Barrett typically obtain values of peak ${ }^{13} \mathrm{CO}$ column densities $\sim 2$ times larger than LKM. Several of these clouds were also observed by Arquilla and Goldsmith (1985). The agreement between Arquilla and Goldsmith and Martin and Barrett (1978) is excellent for the two clouds they have in common, including B227, for which the discrepancy between Martin and Barrett and LKM is most severe. There are also two clouds in common between LKM and Arquilla and Goldsmith (1985): the latter derive ${ }^{13} \mathrm{CO}$ column densities $\sim 3$ times larger than LKM. Although both Martin and Barrett (1978) and Arquilla and Goldsmith (1985) corrected their antenna temperatures for coupling of the beam to the source, whereas LKM did not, these clouds have such large angular sizes that this makes a difference in antenna temperatures of only $\sim 20 \%$ in the worst case. The main cause of the discrepancy is the fact that LKM derived their column densities using the maximum values found in the cloud of $T_{R}^{*}$ for both transitions. Since the largest values of $T_{R}^{*}\left({ }^{13} \mathrm{CO}\right)$ are often found off of the peak in ${ }^{12} \mathrm{CO}$ (see, e.g., the maps in Martin and Barrett 1978), this procedure tends to systematically understimate the column densities of ${ }^{13} \mathrm{CO}$.

I have therefore revised the mass and density estimates for the clouds in the sample of LKM; the adopted values are given in Table 1. Where observations from Martin and Barrett (1978) or Arquilla and Goldsmith (1985) are available, these have been used to derive cloud properties. Since the cloud masses have been lowered by about an order of magnitude compared to the values given by LKM, it is evident that the behavior of mean density with radius in these dark clouds cannot represent a continuation of the trend in dense cloud cores; indeed, since

TABLE 1

ReVised Parameters For LKM Dark Clouds

\begin{tabular}{|c|c|c|c|c|c|}
\hline Object & $\begin{array}{c}R \\
(\mathrm{pc})\end{array}$ & $\begin{array}{c}M_{c}^{\mathrm{a}} \\
\left(M_{\odot}\right)\end{array}$ & $\begin{array}{l}\left\langle n\left(\mathrm{H}_{2}\right)\right\rangle \\
\left(\mathrm{cm}^{-3}\right)\end{array}$ & $\underset{(\mathrm{pc})}{D}$ & Source \\
\hline IC $1848-1$. & 12. & 9100. & 20. & 1700 & MB \\
\hline & 1.4 & 87. & 120. & 160 & LKM \\
\hline ORI-I-2 .. & 0.35 & 4.0 & 350. & 400 & $\mathrm{MB}$ \\
\hline B34 $\ldots \ldots \ldots$ & 1.3 & 8.6 & 15. & 200 & LKM \\
\hline $\mathrm{L} 1672 \ldots .$. & 1.2 & 59. & 130. & 200 & LKM \\
\hline B227 ....... & 0.9 & 28. & 145. & 400 & AG \\
\hline B255 ........ & 0.52 & 0.33 & 9. & 200 & LKM \\
\hline B68 ........ & 0.19 & 0.58 & 320. & 200 & MB \\
\hline B118 ...... & 0.38 & 0.73 & 50. & 400 & MB \\
\hline B133 ........ & 1.8 & 18. & 12. & 400 & LKM \\
\hline B134 ........ & 0.93 & 13. & 61. & 400 & LKM \\
\hline B335 ....... & 073 & 16.8 & 160. & 250 & MB \\
\hline B361 ...... & 1.62 & 98. & 90. & 350 & AG \\
\hline B362 ....... & 3.9 & 120. & 8. & 200 & LKM \\
\hline B157 ........ & 2.7 & 74. & 14. & 400 & LKM \\
\hline B161 ....... & 1.6 & 23. & 21. & 150 & LKM \\
\hline
\end{tabular}

a Mass including correction for $\mathrm{He}$.

SOURCES.-MB, Martin and Barrett 1978; AG, Arquilla and Goldsmith 1985; LKM, Leung, Kutner, and Mead 1982. most of the range in cloud size in the data used by Myers (1983) comes from the LKM sample, it is no longer clear that the dense cloud cores show any evidence at all for $\bar{n} \propto r^{-1}$ or $\Delta V \propto r^{1 / 2}$.

In Figure $1 \mathrm{I}$ have plotted mean $\mathrm{H}_{2}$ density versus radius for 30 dark clouds, with data from LKM, Arquilla and Goldsmith (1985), Martin and Barrett (1978), and Snell (1981). Also plotted are data for 18 high-latitude clouds observed by Keto and Myers (1986). Superposed on the data are the predicted $n-r$ relations for pressure-bounded polytropes, for pressures $P / k=10^{4}$ and $10^{3}$, for a polytropic index of $N=-3$ and two values of $\xi_{e}: 0.5$ (corresponding to a nearly uniform density cloud) and 20 (a very centrally condensed cloud). Considering the simplifying assumptions made in the models (spherical clouds, the assumption that the polytropes are complete polytropes, the uncertain nature of "turbulence" in clouds, neglect of magnetic fields and rotation) and the uncertainties in deriving sizes and mean densities from molecular observations, the agreement with observations is quite good. It is also evident that most of the objects from Keto and Myers are best represented by $\xi_{e}=0.5$ models; i.e., self-gravity is not very important for these objects. This conclusion was reached by Keto and Myers, who suggested that the high-latitude clouds which they observed must be pressure-confined, with an intercloud medium at $P / k=3 \times 10^{3}-3 \times 10^{4}$, a result in good agreement with the present work. (Keto and Myers obtained somewhat larger values for the boundary pressure than inferred here because they assumed uniform clouds.) The three clouds which lie substantially above the $\xi_{e}=20, P / k=10^{4}$ line are L134N and L1551 (from Snell 1981) and CRL 437 (from Arquilla and Goldsmith 1985) which are all exceptional dark globules in that they are forming stars. However, B335, which also has an embedded infrared source, does not stand out. Frerking, Langer, and Wilson (1986) have observed B335 extensively with the Bell Labs $7 \mathrm{~m}$ telescope and concluded that it is much more complex than revealed by the data of Martin and Barrett (1978), which are used here. It is quite likely that the masses of many of these clouds have been underestimated, due to destruction of $\mathrm{CO}$ in the high-latitude clouds and to subthermal excitation of optically thick ${ }^{13} \mathrm{CO}$ in the dark clouds.

Figure 2 shows the line width-radius data for the clouds from LKM $\left({ }^{13} \mathrm{CO}\right.$ line widths) and Keto and Myers (1986) $\left({ }^{12} \mathrm{CO}\right.$ line widths) (line widths were not available for the other clouds in the sample), along with the corresponding predictions for pressure-bounded polytropes, for the same parameters as in Figure 1. The predicted line widths have been scaled by a factor $\alpha=1.4$ as an approximate saturation correction; this should be a reasonable value for both the LKM data and the Keto and Myers observations, since the ${ }^{13} \mathrm{CO}$ lines probably have moderate optical depths and the column densities of ${ }^{12} \mathrm{CO}$ in the high-latitude clouds are considerably smaller than those in typical dark clouds, and are of the same order as the ${ }^{13} \mathrm{CO}$ column densities of the LKM clouds. The agreement is again quite good, and many of the Keto and Myers clouds appear to be quite uniform in structure as noted above. Including the size-line width data of Myers and Benson (1982) shows that the dense cores do appear to continue the trend of line width with size shown by the dark clouds (see Fig. 1 of Myers 1983), but it is possible that comparing them is inherently unfair, since the dense cores are probably dominated by selfgravity. On the other hand, it is possible that the apparent continuity seen here reflects the same physics, namely, the influence of the boundary pressure on the cloud structure. In 


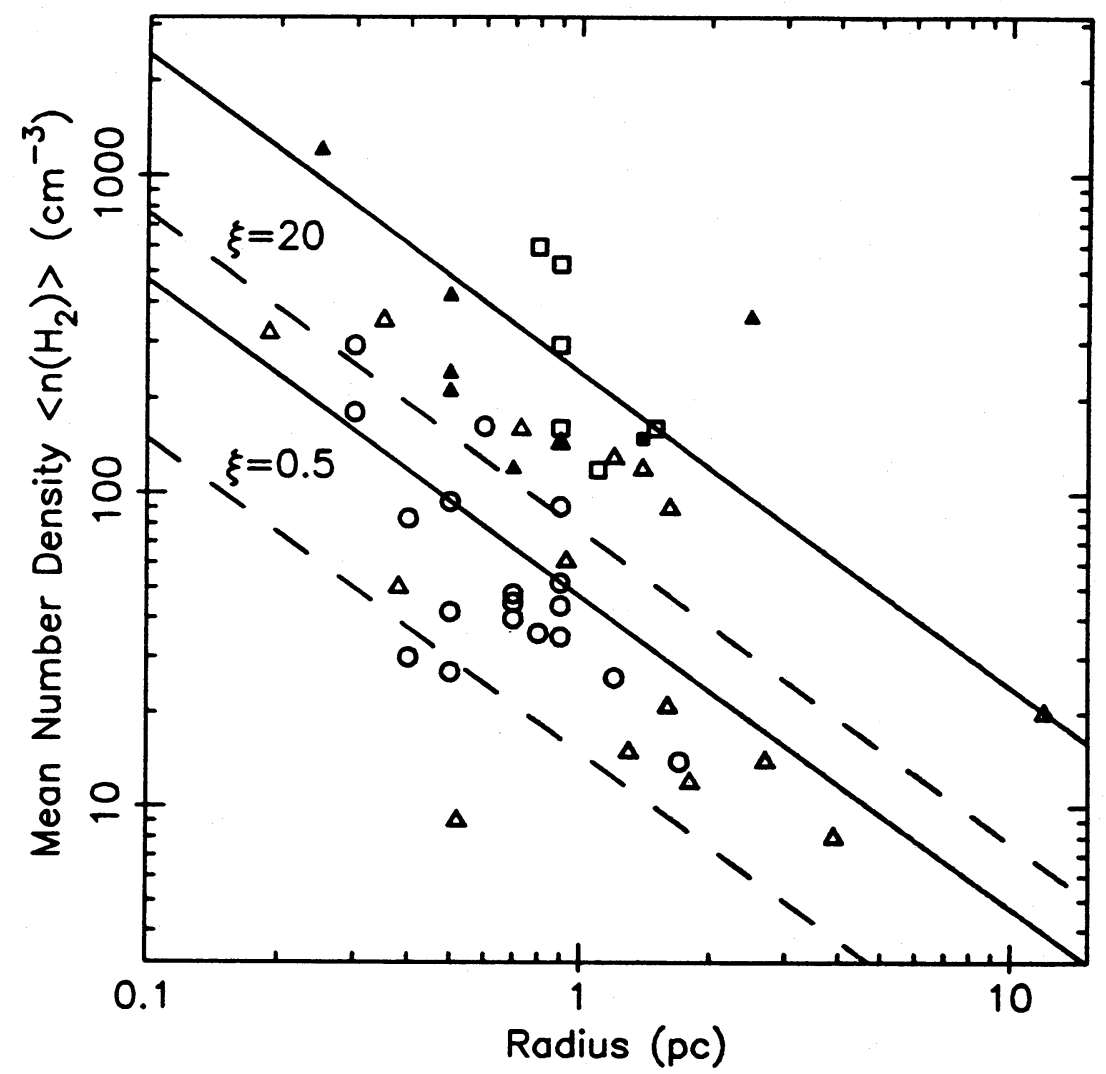

FIG. 1.- Mean number density vs. radius for dark clouds and high-latitude clouds. Triangles are from Leung, Kutner, and Mead (1982), revised as described in the text; filled triangles are from Arquilla and Goldsmith (1985); squares are from Snell (1981); filled square is from Martin and Barrett (1978); circles are from Keto and Myers. Dashed lines are the mean number density-size relations predicted for pressure-bounded clouds for $P / k=10^{3}$, and are labeled with the value of $\xi_{e}$; solid lines are for $P / K=10^{4}$ and are ordered by $\xi_{e}$ in the same way.

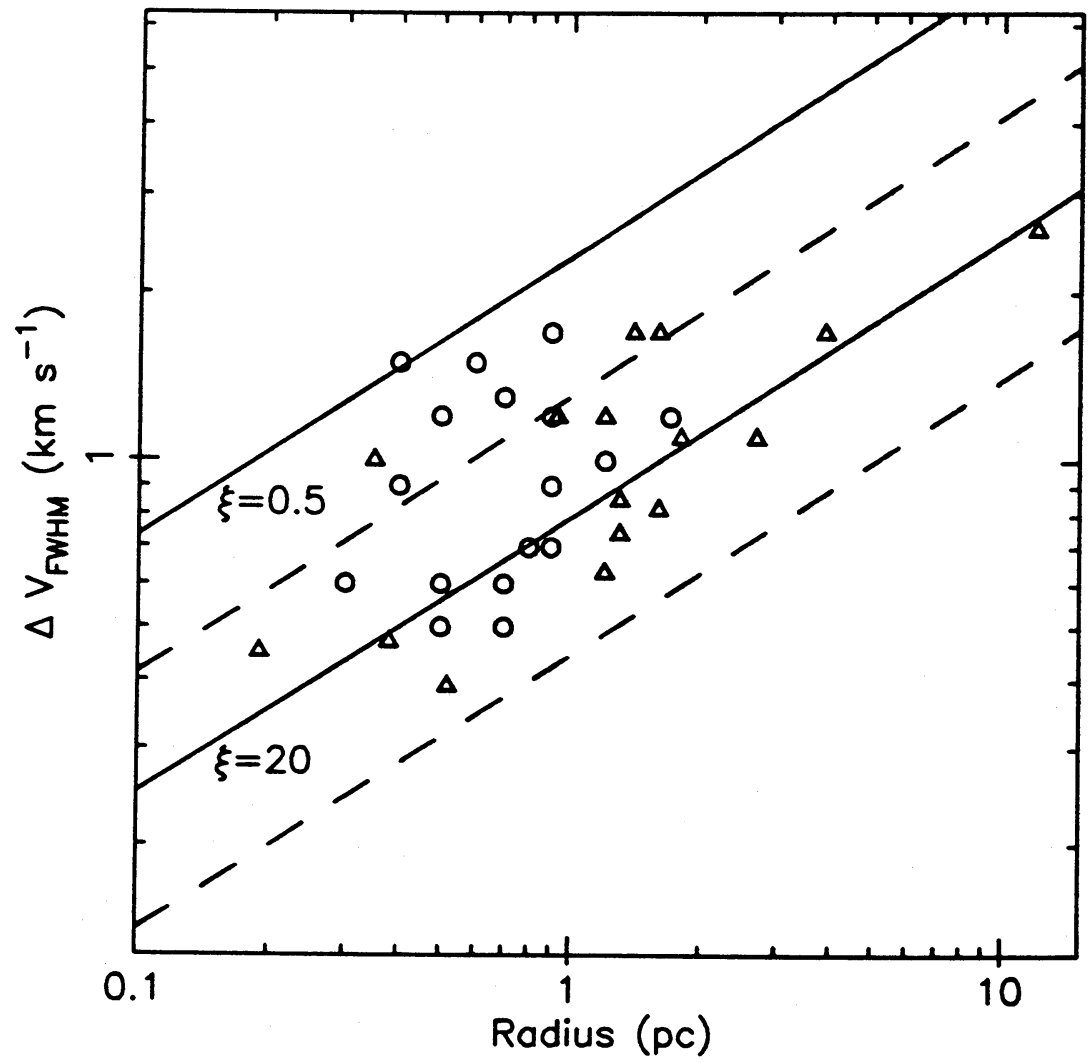

Fig. 2.-Line width vs. radius for the dark globules of Leung, Kutner, and Mead (1982) and the high-latitude clouds of Keto and Myers (1986). Data symbols and predicted values for polytrope models as in Fig. 1 . 
the case of the dense cores, the boundary pressure presumably represents that of the surrounding molecular gas, rather than the intercloud medium.

Table 2 lists the properties of pressure-bounded polytropes for a range of indices for a boundary pressure of $P / k=10^{4}$ and a central number density of $\mathrm{H}_{2}$ of $3000 \mathrm{~cm}^{-3}$. The models with large negative indices are more centrally concentrated, with the result that their total internal energies

$$
U \equiv \int_{0}^{M} c_{v} T d m=\frac{1}{\gamma-1} \int p d V
$$

(where $c_{v}$ is the specific heat per gram at constant volume and $\gamma$ the ratio of specific heats at constant pressure and volume) exceed their gravitational energies

$$
W \equiv-G \int_{0}^{M} m d m / r
$$

by only a small factor, unlike the small-index models where $U$ can exceed $W$ by nearly an order of magnitude. ( $U$ and $W$ are calculated using the equations given in Viala and Horedt 1974b.) For a given boundary pressure and polytropic index, the internal energy per unit volume averaged over a cloud is constant along the equilibrium sequence of increasing central density and decreasing radius.

\section{IMPLICATIONS}

As we have seen above, pressure-bounded polytropes predict the observed size-line width relations and inferred mean density-size relations found in dark clouds. A "universal" value of the conversion factor relating integrated ${ }^{12} \mathrm{CO}$ line intensity, $I_{\mathrm{CO}}$, to $\mathrm{H}_{2}$ column density, is usually considered to be a consequence of self-gravitating, "virialized" clouds (Dickman, Snell, and Schloerb 1986; Solomon et al. 1986), where the term "virialized" is here used in the sense defined in $\S \mathrm{I}$, to mean that the velocity dispersion $\sigma$ is determined directly by the cloud mass and radius (see Maloney and Black 1988). It is of interest to derive a corresponding relation for pressure-bounded clouds. The cloud-averaged column density of $\mathrm{H}_{2}$ is given by

$$
N\left(\mathrm{H}_{2}\right)=1.20 \times 10^{19}\left[\frac{-(N+1)}{\theta_{e}^{N+1}}\left(\frac{P_{e}}{k}\right)\right]^{1 / 2} \theta_{e}^{\prime} \mathrm{cm}^{-2} .
$$

Note that this is the true column density of molecular hydrogen, not the column density of particles of mean mass. The integrated $\mathrm{CO}$ intensity averaged over the cloud can be approximated by

$$
I_{\mathrm{CO}}=\alpha_{s}(2 \pi)^{1 / 2} T_{A} \sigma_{v}
$$

where $\sigma_{v}$ is the one-dimensional velocity dispersion, $T_{A}$ is the antenna temperature at line center averaged over the cloud, and $\alpha_{s}$ depends on the degree of line saturation. For realistic cloud parameters, $\alpha_{s}$ is probably in the range $1.5-2.0$ for ${ }^{12} \mathrm{CO}$ (Kleiner and Dickman 1985). I will adopt $\alpha_{s}=1.75$ and use the cloud center value of $\sigma_{v}$. Equations (6), (8), and (9) imply that the cloud-averaged value of $N\left(\mathrm{H}_{2}\right) / I_{\mathrm{CO}}$ is

$$
\frac{\bar{N}\left(\mathrm{H}_{2}\right)}{I_{\mathrm{CO}}}=4.77 \times 10^{19}\left(\frac{\xi_{e}}{r_{e}}\right)^{1 / 2} \frac{\theta_{e}^{\prime}}{T_{A}}\left[-(N+1)^{3} \frac{P_{e} / k}{\theta_{e}^{N+1}}\right]^{1 / 4},
$$

with $I_{\mathrm{Co}}$ in $\mathrm{K} \mathrm{km} \mathrm{s}^{-1}$ and $r_{e}$ in parsecs. A value for $T_{A}=6.7 \mathrm{~K}$ corresponds to optically thick $\mathrm{CO}$ at an excitation temperature of $10 \mathrm{~K}$. With this and $P_{e} / k=10^{4}, \xi_{e}=20$, and $N=-3$,

$$
\bar{N}\left(\mathrm{H}_{2}\right) / I_{\mathrm{CO}}=1.4 \times 10^{20} r_{e}^{-1 / 2}
$$

in units of $\mathrm{cm}^{-2}\left(\mathrm{~K} \mathrm{~km} \mathrm{~s}^{-1}\right)^{-1}$. Thus $\bar{N}\left(\mathrm{H}_{2}\right) / I_{\mathrm{CO}}=2.0 \times 10^{20}$ for $r_{e}=0.5 \mathrm{pc}$, while for $r_{e}=5 \mathrm{pc}$ it has fallen only to $6.4 \times 10^{19}$. Note that for the small clouds the line width for molecules like $\mathrm{CO}$ is overestimated, while the cloud-averaged value will substantially underestimate $N\left(\mathrm{H}_{2}\right) / I_{\text {co }}$ for resolved clouds. The ratio of the cloud center column density to the cloud-averaged column density for a polytropic cloud model is given by

$$
\frac{N\left(\mathrm{H}_{2}\right)_{\mathrm{cent}}}{\bar{N}\left(\mathrm{H}_{2}\right)}=\frac{\int_{0}^{\xi_{e}} \theta^{N} d \xi}{2 \theta_{e}^{\prime}} .
$$

For $N=-3$, this ratio is equal to 3.4 and 8.7 for $\xi_{e}=5$ and 20 , respectively. Thus for clouds which are substantially resolved by the antenna beam, such as those in the LKM sample, the appropriate predicted value of $N\left(\mathrm{H}_{2}\right) / I_{\mathrm{CO}}$ is a few times larger than the value given by equation (11).

This number can be compared with the value derived from large-scale gamma-ray studies by Bloemen et al. (1986), which is $(2.8 \pm 1.0) \times 10^{20}$. Equations (11) and (12) show that the predicted $N\left(\mathrm{H}_{2}\right) / I_{\mathrm{Co}}$ for the model clouds is in good agreement for the small clouds ( $\lesssim 5 \mathrm{pc}$ ), while it is too small for the larger clouds. Of course, it is well known that the large molecular clouds do not have smooth density profiles, but are extremely clumpy on small scales. One could thus imagine complexes made up of small pressure-bounded clumps embedded in a lower density envelope in pressure equilibrium with the intercloud medium. Such a scenario was suggested by Elmegreen (1985), although he considered self-gravitating clumps within lower density, self-gravitating envelopes in pressure equilibrium with the intercloud medium. Systems like giant molecular cloud complexes, with large masses in clumps, would probably be gravitationally bound in any case. However, one must be extremely cautious in interpreting conventional plots of virial mass versus CO luminosity, since the observed correlation of these quantities is solely an artifact of the line width-

TABLE 2

Parameters of Pressure-bounded Clouds

\begin{tabular}{cccccccc}
\hline \hline Index & $\begin{array}{c}\text { Radius } \\
(\mathrm{pc})\end{array}$ & $\begin{array}{c}\text { Mass } \\
\left(M_{\odot}\right)\end{array}$ & $\begin{array}{c}\bar{n}\left(\mathrm{H}_{2}\right) \\
\left(\mathrm{cm}^{-3}\right)\end{array}$ & $\begin{array}{c}T_{0} \\
(\mathrm{~K})\end{array}$ & $\begin{array}{c}\Delta V_{0} \\
\left(\mathrm{~km} \mathrm{~s}^{-1}\right)\end{array}$ & $\begin{array}{c}U \\
(\mathrm{ergs})\end{array}$ & $\begin{array}{c}W \\
(\mathrm{ergs})\end{array}$ \\
\hline$-1.5 \ldots \ldots \ldots$ & 0.80 & 23.6 & 171. & 8.0 & 0.11 & $1.53 \times 10^{44}$ & $-4.09 \times 10^{43}$ \\
$-2.0 \ldots \ldots \ldots$ & 1.73 & 148. & 108. & 18.5 & 0.43 & $1.71 \times 10^{45}$ & $-7.79 \times 10^{44}$ \\
$-3.0 \ldots \ldots \ldots$ & 4.44 & 1248. & 54.0 & 60.7 & 1.02 & $3.37 \times 10^{46}$ & $-2.29 \times 10^{46}$ \\
$-4.0 \ldots \ldots \ldots$ & 8.16 & 4843. & 33.8 & 137. & 1.61 & $2.36 \times 10^{47}$ & $-1.95 \times 10^{47}$ \\
$-5.0 \ldots \ldots \ldots$ & 12.7 & 12700. & 23.4 & 249. & 2.21 & $9.66 \times 10^{47}$ & $-8.87 \times 10^{47}$ \\
\hline
\end{tabular}

NoTE.-Properties of pressure-bounded polytropes: $P / k=10^{4} ; n_{0}\left(\mathrm{H}_{2}\right)=3000$. 
size relation which is seen for giant molecular clouds as well as dark clouds.

Most analyses of the stability of molecular clouds have ignored the role of the boundary pressure. Exceptions are the work of Elmegreen (1985), referred to above, Dickman and Clemens (1983), and Chieze (1987). Elmegreen specifically considered the role of magnetic fields, and from a viral theorem analysis obtained the result $\Delta V \propto r^{1 / 2}$. More directly related to this work is the analysis of Dickman and Clemens (1983), who considered pressure-bounded isothermal and polytropic models for a low-mass, very quiescent Bok globule, and concluded that it could well be in a state of hydrostatic equilibrium. Dickman and Clemens used a smaller value for the pressure in the interstellar medium $\left(P / k=5 \times 10^{3}\right)$ and found no reason to appeal to magnetic support to stabilize the cloud, based on the derived cloud temperature and mass. Adopting a value for the pressure 2 or 3 times higher might alter that conclusion. Finally, it is important to call attention to the work of Chieze (1987) (first referenced in Silk 1985), who was the first to point out that if molecular clouds are in equilibrium with an external pressure, then the expected scaling of velocity dispersion with cloud radius and intercloud pressure is $\sigma \propto r^{1 / 2} P^{1 / 4}$.

The predominance of pressure-bounded clouds has a number of interesting implications for the ISM. If we identify these clouds with dark clouds, it implies that they are inherently stable and are not likely to form stars unless exposed to an increase in intercloud pressure which compresses them enough for gravitational forces to dominate; this might result from a supernova blast wave, for example. Star formation would then be a consequence of the collection of these clouds into large complexes which are gravitationally bound. In a very actively star-forming environment such as a starburst galaxy, the increase of pressure in the interstellar medium caused by the presence of many early-type stars might trigger the widespread collapse of such objects.

The success of the pressure-bounded cloud model in explaining the properties of dark clouds suggests that these clouds may be quite distinct from the larger molecular cloud complexes. The size-line width relation of Solomon et al. (1986) for a large sample of giant molecular clouds predicts lines that are too broad at size scales corresponding to the dark clouds ( $\approx 1 \mathrm{pc}$ and less). This lack of continuity may represent a transition from pressure-bounded to gravitationally bound clouds. However, the fact that the giant molecular clouds also display a relation of the same form as predicted for pressure-bounded clouds suggests that the effect of the intercloud pressure on cloud complexes may still have important consequences. It should be noted that Roberts and Stewart (1987), in emphasizing the possible importance of orbit crowding for the formation of giant molecular cloud complexes in galaxies with spiral density waves, suggest that a substantially smaller fraction of such cloud complexes may be self-gravitating than has previously been thought.

A few cautionary remarks should be made here. The line widths of most molecular clouds are considerably in excess of thermal, so that justification of a polytropic equation of state on the basis of thermal balance calculations is not really viable. However, whatever process produces the turbulence observed in the ISM may lead to an equation of state that can be reasonably described by a polytropic law if the heating and dissipation rates have an appropriate dependence on cloud density. If, for example, we regard the observed cloud velocity dispersions as arising from the motions of clumps within the cloud envelope, then the rate of energy dissipation per unit volume due to clump collisions is given by

$$
\Lambda_{c}=C M_{c} v_{\mathrm{rms}}^{2} n_{c}^{2} \sigma_{\mathrm{geom}} v,
$$

where $v_{\text {rms }}$ is the one-dimensional clump velocity dispersion, $v$ is the relative velocity between clumps, $M_{c}$ the mass of the clumps (assumed identical), $n_{c}$ is the number density of clumps, $\sigma_{\text {geom }}$ is the geometric clump cross section, and $C$ is a constant of order unity (Scalo and Pumphrey 1982; Elmegreen 1985). This expression holds whether magnetic fields are present or not; only the value of the constant $C$ is affected (Elmegreen 1985). Since the dark clouds studied by Arquilla and Goldsmith (1985) are strongly centrally condensed, equation (13) indicates that the dissipation rate will increase rapidly toward the cloud center. If whatever mechanism powers the turbulence does not have an equally strong dependence on clump density (equal to the mass density if the clumps dominate the cloud mass), then it is to be expected that the velocity dispersion will decline toward the cloud center, and the equation of state describing the cloud may resemble a polytropic one. Unfortunately, our understanding of turbulence in the interstellar medium is so inadequate that it is very difficult to quantify this further (see Scalo 1987).

Magnetic fields have not been specifically included in the present work; a realistic cloud model should discuss them explicitly and relax the assumption of spherical symmetry. In addition to the work of Elmegreen (1985), important papers dealing with the possible role of magnetic fields in clouds are Myers (1987) and especially Falgarone and Puget (1986).

In summary, the sizes, masses, and predicted $\mathrm{CO}$ emission from negative-index polytropic cloud models in equilibrium with external pressures $P / k \gtrsim 10^{4}$ are in good agreement with the properties of dark clouds. Furthermore, the pressure bounded clouds are predicted to have size-line width and mean density-size relations of the same form and absolute value as have been observed and inferred for dark clouds. Thus the observed features of these interstellar clouds do not require that they be completely self-gravitating.

I would like to thank John Black for valuable comments on a draft of this paper, as well as for providing data in advance of publication. I would also like to thank the referee for a number of insightful comments, and for providing several important references. This work was supported by NASA Theoretical Astrophysics grant NAGW-763 to the University of Arizona.

\section{APPENDIX STABILITY OF NEGATIVE-INDEX POLYTROPES WITH CONSTANT CENTRAL TEMPERATURES}

The general properties of negative-index polytropes are extensively discussed by Viala and Horedt (1974a, $b$ ). In their second paper, Viala and Horedt discuss the stability of negative-index polytropes and demonstrate that they are unstable for values of the dimensionless radius $\xi$ in excess of a critical value which is a function of the polytropic index, $N$. Here I consider the stability of such polytropes to external perturbations, under the constraint that their central temperatures remain constant. 
It is easy to see that a polytropic gas sphere will be unstable if $(\partial P / \partial V)_{0}$ is positive, where the subscript 0 denotes that the derivative is evaluated at the boundary of the polytrope. If an external perturbation such as an increase in the bounding pressure is applied to such a polytrope, the corresponding decrease in the volume of the sphere will result in a fruther decrease in the surface pressure, with the result that the polytrope will contract still further. We wish to derive an expression for $(\partial P / \partial V)_{0}$ applicable to a polytrope undergoing such a perturbation, which can then be evaluated to determine its stability according to this criterion.

Following Bonnor (1958), we will consider a spherical mass of gas in equilibrium, with density $\rho(r)$ and pressure $P(r)$ at radius $r$. The gas is assumed to have spherical symmetry, so that the equation of hydrostatic equilibrium is

$$
\frac{d P(r)}{d r}=-\rho(r) \frac{G M(r)}{r^{2}}
$$

where $M(r)$ is the mass inside radius $r$. Assuming that the gas is polytropic, it will obey the equation of state

$$
P=K \rho^{1+1 / N} \text {. }
$$

Since we are requiring the central temperature of the cloud $T_{c}$ to stay constant, the polytropic constant $K$ is not invariant if the sphere is subjected to an external perturbation, since

$$
K=\frac{R T_{c} \rho_{c}^{-1 / N}}{\mu},
$$

where $\rho_{c}$ is the central density of the polytrope, $R$ is the gas constant, and $\mu$ is the molecular weight of the gas. Along the equilibrium sequence of constant $K, T_{c}$ must decrease as the central density increases, and vice versa. We therefore define a new constant $K^{\prime}=R T_{c} / \mu$, so that the polytropic equation of state under the restriction of constant $T_{c}$ is

$$
P=K^{\prime} \rho_{c}^{-1 / N} \rho^{1+1 / N} .
$$

Imagine a sphere with radius $r_{0}$ concentric with the polytrope boundary. The quantity $r_{0}$ may or may not be coincident with the radius of the polytrope. This sphere contains a mass of gas $M$. We will allow $r_{0}$ to vary by a small amount, subject to the constraints: (1) $M$ stays constant; (2) Equation (A1) remains satisfied throughout; (3) Equation (A4) is satisfied during the variation. We will also assume that the pressure at the new value of $r_{0}$ is that appropriate for equilibrium.

The usual polytropic variables $\theta$ and $\xi$ are introduced through the formulae

where

$$
\rho=\rho_{c} \theta^{N}, \quad \xi=r / \alpha,
$$

$$
\alpha=\left[-\frac{K(1+N)}{4 \pi G \rho_{c}^{(1-1 / N)}}\right]^{1 / 2} .
$$

Since $\alpha$ will not be constant as the sphere is perturbed, we will define the new variable

then

$$
\gamma=\left[-\frac{K^{\prime}(1+N)}{4 \pi G}\right]^{1 / 2}
$$

$$
\alpha=\gamma \rho_{c}^{-1 / 2} .
$$

The structure of a polytrope is determined by the solution to the Lane-Emden equation of index $N$,

$\theta(\xi)$ is also constrained to satisfy

$$
\frac{1}{\xi^{2}} \frac{d}{d \xi}\left(\xi^{2} \frac{d \theta}{d \xi}\right)=\theta^{N}
$$

$$
\theta(0)=1, \quad \frac{d \theta(0)}{d \xi}=0 .
$$

The mass of the polytrope within radius $r_{0}$ can then be written as

$$
M=4 \pi \gamma^{3} \rho_{c}^{-1 / 2} \xi_{0}^{2}\left(\frac{d \theta}{d \xi}\right)_{0}
$$

(Viala and Horedt 1974b), where the subscript 0 refers to the values at $r=r_{0}$ (and thus $\xi=\xi_{0}$ ). Since the mass must remain constant,

$$
\delta M=0=4 \pi \gamma^{3} \rho_{c}^{-1 / 2} \frac{d}{d \xi}\left(\xi^{2} \frac{d \theta}{d \xi}\right)_{0} \delta \xi_{0}-2 \pi \gamma^{3} \rho_{c}^{-3 / 2} \xi_{0}^{2}\left(\frac{d \theta}{d \xi}\right)_{0} \delta \rho_{c} .
$$

Using equations (A5) and (A8), we can express $\delta \xi_{0}$ as

$$
\delta \xi_{0}=\frac{1}{\gamma \rho_{c}^{-1 / 2}} \delta r_{0}+\frac{r_{0} \rho_{c}^{-1 / 2}}{2 \gamma} \delta \rho_{c}
$$


Substituting equation (A13) into equation (A12) and simplifying the resulting expression using equations (A9) and (A11) yields

$$
4 \pi \gamma^{2} \frac{\rho_{0}}{\rho_{c}} \xi_{0}^{2}\left(\delta r_{0}+\frac{r_{0}}{2 \rho_{c}} \delta \rho_{c}\right)+\frac{1}{2} M \rho_{c}^{-1} \delta \rho_{c}=0
$$

where $\rho_{0}=\rho\left(\xi_{0}\right)$. Equation (A14) can be solved for $r_{0}$ and further simplified by noting that $\gamma^{2}=\alpha^{2} \rho_{c}$, so that

$$
\delta r_{0}=\left(\frac{M}{8 \pi r_{0}^{2} \rho_{0} \rho_{c}}-\frac{r_{0}}{2 \rho_{c}}\right) \delta \rho_{c} .
$$

We now need an expression for $\delta P_{0}$. As noted above, under the constraint of $T_{c}=$ constant we must write the polytropic equation of state in the form (A4), so that

$$
\delta P=K^{\prime}\left(-\frac{1}{N}\right) \rho_{c}^{-1 / N-1} \rho^{(1+1 / N)} \delta \rho_{c}+K^{\prime} \rho_{c}^{-1 / N}\left(1+\frac{1}{N}\right) \rho^{1 / N} \delta \rho=\left(-\frac{1}{N}\right) \frac{P}{\rho_{c}} \delta \rho_{c}+\left(1+\frac{1}{N}\right) \frac{P}{\rho} \delta \rho .
$$

Hence

$$
\frac{\delta P}{P}=\left(-\frac{1}{N}\right) \frac{\delta \rho_{c}}{\rho_{c}}+\left(1+\frac{1}{N}\right) \frac{\delta \rho}{\rho} .
$$

Using the defining equation (A5) for $\theta$, we can expand the term $\delta \rho$ as $\delta \rho=\delta \rho_{c} \theta^{N}+\rho_{c} N \theta^{N-1} \delta \theta$, so that

$$
\frac{\delta \rho}{\rho}=\frac{\delta \rho_{c}}{\rho_{c}}+\frac{N}{\theta} \delta \theta
$$

Substituting into equation (A17),

$$
\frac{\delta P}{P}=\frac{\delta \rho_{c}}{\rho_{c}}+\frac{N}{\theta} \delta \theta
$$

An expression for $\delta \theta_{0}=(d \theta / d \xi)_{0} \delta \xi_{0}$ can be obtained from equations (A11) and (A13), which after simplifying as in equation (A15) is

$$
\delta \theta_{0}=\frac{M}{4 \pi \gamma^{2} r_{0}^{2}} \delta r_{0} r_{0}+\frac{M}{8 \pi \gamma^{2} r_{0} \rho_{c}} \delta \rho_{c} .
$$

Putting this expression for $\delta \theta_{0}$ into equation (A19) and using equation (A15) for $\delta r_{0}$, we obtain after some algebra

$$
\frac{\delta P_{0}}{\delta r_{0}} \frac{1}{P_{0}}\left(M-4 \pi r_{0}^{3} \rho_{0}\right)\left(4 \pi \gamma^{2} r_{0}^{2}\right)=32 \pi^{2} \gamma^{2} r_{0}^{4} \rho_{0}+(N+1)\left(\frac{\rho_{c}}{\rho_{0}}\right)^{1 / N} M^{2}
$$

This is nearly the desired expression; we merely have to arrange it into a more convenient form. If we divide by $4 \pi \gamma^{2} r_{0}^{2}$, then the right-hand side becomes

$$
8 \pi r_{0}^{2} \rho_{0}+(N+1)\left(\frac{\rho_{c}}{\rho_{0}}\right)^{1 / N} \frac{M^{2}}{4 \pi \gamma^{2} r_{0}^{2}} .
$$

Making use of the definitions of $\gamma$ and $K^{\prime}$, the second term on the right-hand side may be written as $-\left(G M^{2} / P_{0} r_{0}^{2}\right) \rho_{0}$, and so the right-hand side can be written

$$
8 \pi r_{0}^{2} \rho_{0}\left(1-\frac{G M^{2}}{8 \pi P_{0} r_{0}^{4}}\right) .
$$

The term in parentheses on the left-hand side may be rearranged as

so that equation (A21) becomes

$$
4 \pi r_{0}^{3} \rho_{0}\left(\frac{M}{4 \pi r_{0}^{3} \rho_{0}}-1\right)
$$

$$
\frac{1}{P_{0}} \frac{\delta P_{0}}{\delta r_{0}}=\frac{2}{r_{0}} \frac{\left[1-\left(G M^{2} / 8 \pi P_{0} r_{0}^{4}\right)\right]}{\left[\left(M / 4 \pi r_{0}^{3} \rho_{0}\right)-1\right]},
$$

which, in the limit of infinitesimal $\delta P_{0}, \delta r_{0}$ becomes

$$
\left(\frac{\partial P}{\partial r}\right)_{0}=-\frac{2}{3} \frac{P_{0}}{r_{0}} \frac{\left[1-\left(G M^{2} / 8 \pi P_{0} r_{0}^{4}\right)\right]}{\left[1-\left(M / 3 V_{0} \rho_{0}\right)\right]},
$$

where we have also made use of $V_{0}=(4 \pi / 3) r_{0}^{3}$. 
From the chain rule,

$$
\left(\frac{\partial P}{\partial V}\right)=\left(\frac{\partial P}{\partial r}\right)\left(\frac{\partial r}{\partial V}\right)=\left(\frac{\partial P}{\partial r}\right)\left(\frac{3}{4 \pi}\right)^{1 / 3} \frac{1}{3} V_{0}^{-2 / 3}
$$

Also $r_{0}=(3 / 4 \pi)^{1 / 3} V_{0}^{1 / 3}$, and so

$$
\left(\frac{\partial P}{\partial V}\right)_{0}=-\frac{2}{3} \frac{P_{0}}{V_{0}} \frac{\left[1-\left(G M^{2} / 8 \pi P_{0} r_{0}^{4}\right)\right]}{\left[1-\left(M / 3 V_{0} \rho_{0}\right)\right]} .
$$

Note that all explicit dependence on the polytropic index has been lost.

In order for polytropes with constant $T_{c}$ to be unstable, the term

$$
\frac{\left(M / 4 \pi r_{0}^{3} \rho_{0}\right)-1}{1-\left(M / 3 V_{0} \rho_{0}\right)}
$$

must be less than zero. Thus if the numerator is positive, the denominator must be negative, and vice versa. Does this occur for any values of $\xi$ ?

To determine whether these polytropes are stable or not, we must examine the behavior of the terms $M / 4 \pi r_{0}^{3} \rho_{0}$ and $M / 3 V_{0} \rho_{0}$ as functions of $\xi$ for different values of the polytropic index. Consider first $M / 3 V_{0} \rho_{0}$. We can write the mass of the polytrope as $M=V_{0} \bar{\rho}$, where $\bar{\rho}$ is the volume-averaged density. Using equations (A7) and (A11), one can show that

$$
\bar{\rho}=3 \rho_{c} \theta_{0}^{\prime} / \xi_{0} \text {. }
$$

Thus we can write

$$
\frac{M}{3 V_{0} \rho_{0}}=\frac{\bar{\rho}}{3 \rho_{0}}=\frac{\rho_{c} \theta_{0}^{\prime}}{\rho_{0} \xi_{0}}=\frac{\theta_{0}^{\prime}}{\xi_{0} \theta_{0}^{n}},
$$

using equation (A5), the definition of $\theta$.

The maximum value of $\theta_{0}^{\prime} / \xi_{0} \theta_{0}^{n}$ can be evaluated from the tables of Viala and Horedt $(1974 a)$; it increases with increasing $|N|$, but is less than unity for all $N$. Thus in order for any of these polytropes to be unstable, we must have

$$
\frac{M}{4 \pi r_{0}^{3} \rho_{0}}>1
$$

Again writing $M=V_{0} \bar{\rho}$, we can write $M / 4 \pi r_{0}^{3} \rho_{0}$ as

$$
\frac{M}{4 \pi r_{0}^{3} \rho_{0}}=\frac{G M \bar{\rho}}{6 P_{0} r_{0}}
$$

We want to eliminate $M, P_{0}$, and $r_{0}$ from this equation. Using equations (A4), (A8), (A11), (A7), and (A26), we can express $M / 4 \pi r_{0}^{3} \rho_{0}$ in terms of the poltropic variables as

$$
\frac{M}{4 \pi r_{0}^{3} \rho_{0}}=-\frac{(1+n)\left(\theta_{0}^{\prime}\right)^{2}}{2 \theta_{0}^{N+1}}
$$

and so negative-index polytropes with constant $T_{c}$ will be unstable only if

$$
-\frac{(1+N)}{2}\left(\theta_{0}^{\prime}\right)^{2} \theta_{0}^{-(N+1)}>1 .
$$

Once again we can use the tables of Viala and Horedt (1974a) to evaluate these functions. It is simplest to write

$$
\frac{\left(\theta_{0}^{\prime}\right)^{2}}{\theta_{0}^{N+1}}>\frac{-2}{(N+1)}
$$

so that for the various values of $N$, the critical values of $\left(\theta_{0}^{\prime}\right)^{2} / \theta_{0}^{N+1}$ are given in Table 3 .

TABLE 3

\begin{tabular}{cc}
\multicolumn{2}{c}{ Critical Values of $\left(\theta_{0}^{\prime}\right)^{2} / \theta_{0}^{N+1}$} \\
\hline \hline$N$ & {$\left[\left(\theta_{0}^{\prime}\right)^{2} / \theta_{0}^{N+1}\right]_{c}$} \\
\hline$-1.5 \ldots \ldots \ldots$ & 4 \\
$-2.0 \ldots \ldots \ldots \ldots$ & 2 \\
$-3.0 \ldots \ldots \ldots \ldots$ & 1 \\
$-4.0 \ldots \ldots \ldots \ldots$ & $\frac{2}{3}$ \\
$-5.0 \ldots \ldots \ldots \ldots$ & $\frac{1}{2}$ \\
$-10 . \ldots \ldots \ldots \ldots$ & $\frac{2}{9}$ \\
\hline
\end{tabular}


Although $\left(\theta_{0}^{\prime}\right)^{2} / \theta_{0}^{N+1}$ gets closer to the critical value with increasing $|N|$, the criterion (A32) is never satisfied. Therefore the sequences of polytropes with constant $T_{c}$ are unconditionally stable to perturbations in external pressure of the type described at the beginning of the Appendix.

Arquilla, R., and Goldsmith, P. F. 1985, Ap. J., 297, 436.

Black, J. H., and Willner, S. P. 1984, Ap. J., 279, 673.

Bloemen, J. B. G. M., et al. 1986, Astr. Ap. 154, 25.

Bonnor, W. B. 1958, M.N.R.A.S., 118, 523 .

Chaffee, F. H., Jr., and White, R. E. 1982, Ap. J. Suppl., 50, 169.

Chieze, J. P. 1987, Astr. Ap., 171, 225.

Crutcher, R. M. 1985, Ap. J., 288, 604

De Jong, T., Dalgarno, A., and Boland, W. 1980, Astr. Ap., 91, 68

Dickman, R. L. 1978, Ap. J. Suppl., 37, 407.

Dickman, R. L., and Clemens, D. P. 1983, Ap. J., 271, 143.

Dickman, R. L., Snell, R. L., and Schloerb, F. P. 1986, Ap. J., 309, 326

Elmegreen, B. G. 1985, Ap. J., 299, 135.

Falgarone, E., and Puget, J. L. 1986, Astr. Ap., 162, 235.

Hawkins, I., Jura, M., and Meyer, D. M. 1985, Ap. J. (Letters), 294, L131.

Hobbs, L. M. 1974, Ap. J., 191, 395.

Jenkins, E. B., and Shaya, E. J. 1979, Ap. J., 231, 55

Jenkins, E. B., Jura, M., and Loewenstein, M. 1983, Ap. J., 270, 88.

Jura, M. 1975, Ap. J., 197, 581.

1987, in Interstellar Processes, ed. D. J. Hollenbach and H. A. Thron-

son (Dordrecht: Reidel), p. 3.

Keto, E. R., and Myers, P. C. 1986, Ap. J., 304, 466.

Kleiner, S. C., and Dickman, R. L. 1985, Ap. J., 295, 466

Leung, C. M. 1985, in Protostars and Planets II, ed. D. C. Black and M. S.

Matthews (Tucson: University of Arizona Press), p. 104

Leung, C. M., Kutner, M. L., and Mead, K. N. 1982, Ap. J., 262, 583.

\section{REFERENCES}

Maloney, P. R., and Black, J. H. 1988, Ap. J., 325, 389.

Martin, R. N., and Barrett, A. H. 1978, Ap. J. Suppl., 36, 1.

Meyer, D. M., and Jura, M. 1985, Ap. J., 297, 119.

Myers, P. C. 1983, Ap. J., 270, 105.

1987, in Interstellar Processes, ed. D. J. Hollenbach and H. A. Thronson (Dordrecht: Reidel), p. 36.

Myers, P. C., and Benson, P. J. 1983, Ap. J., 266, 309.

Roberts, W. W., and Stewart, G. R. 1987, Ap. J., 314, 10

Scalo, J. M. 1987, in Internal Processes, ed. D. J. Hollenbach and H. A. Thronson (Dordrecht: Reidel), p. 349.

Scalo, J. M., and Pumphrey, W. A. 1982, Ap. J. (Letters), 258, L29.

Shu, F. H., Milione, V., Gebel, W., Yuan, C., Goldsmith, D. W., and Roberts, W. W. 1972, Ap. J., 173, 557 .

Silk, J. 1985, in Birth and Infancy of Stars, ed. R. Lucas, A. Ormont, and R. Stora (Amsterdam: Elsevier), p. 349.

Snell, R. L. 1981, Ap. J. Suppl., 45, 121.

Solomon, P. M., Rivolo, A. R., Mooney, T. J., Barrett, J. W., and Sage, L. J. 1986, in Star Formation in Galaxies, ed. C. Persson (NÄSA Conf. Pub. No. 2466), p. 37.

van Dishoeck, E. F., and Black, J. H. 1986, Ap. J. Suppl., 62, 109.

. 1987, in preparation.

Viala, Y. P. 1972, C.R. Acad. Sci., Paris, 275B, 117.

Viala, Y. P., and Horedt, G. P., 1974a, Astr. Ap. Suppl., 16, 173.

. $1974 b$, Astr. Ap., 33, 195.

Philip Maloney: Sterrewacht Leiden, Postbus 9513, 2300 RA Leiden, The Netherlands 